\title{
ATLAS physics prospects for the upgraded LHC
}

\author{
N. Ilic, on behalf of the ATLAS Collaboration ${ }^{1, a}$ \\ ${ }^{1}$ University of Toronto
}

\begin{abstract}
The 2010-2012 operation period at the LHC has been very successful, including the discovery of a new particle with a mass of about $125 \mathrm{GeV}$ compatible within uncertainties with the Higgs boson predicted by Standard Model. Precise measurements of the properties of this boson, and the discovery of new physics beyond the Standard Model, are primary goals of future running at the LHC. The physics prospects with a proton-proton centre-of-mass energy of $14 \mathrm{TeV}$ are presented for $300 \mathrm{fb}^{-1}$ and $3000 \mathrm{fb}^{-1}$ at the high-luminositiy LHC. The ultimate precision attainable on measurements of the couplings of the $125 \mathrm{GeV}$ particle to elementary fermions and bosons is discussed, as well as perspectives on the searches for partners associated with this new object, predicted by several extensions of the standard theory. Supersymmetry is one of the best motivated and well-studied extensions of the Standard Model. The current searches at the LHC have yielded sensitivity to $\mathrm{TeV}$ scale gluinos and 1 st and 2 nd generation squarks, as well as to 3rd generation squarks and electro-weakinos in the hundreds of $\mathrm{GeV}$ mass range. Benchmark studies are presented to show how these limits can be extended for inclusive strong production of squarks and gluinos, direct production of 3rd generation squarks and weak production of electro-weakinos. A considerable fraction of the parameter space for a wide variety of other models has been probed with the $8 \mathrm{TeV}$ data. The prospects of searches for new heavy bosons and dark matter candidates at $14 \mathrm{TeV}$ are explored here. For all these studies, a parameterised simulation of the upgraded ATLAS detector is used, taking into account the expected pileup conditions.
\end{abstract}

\section{Introduction}

Throughout 2010-2012 the Large Hadron Collider delivered $28.4 \mathrm{fb}^{-1}$ of data while operating at a $\sqrt{s}=7 / 8 \mathrm{TeV}$. The ATLAS detector collected $26.4 \mathrm{fb}^{-1}$ of the total delivered integrated luminosity. Analysis of this data has strengthened the validity of the Standard Model, especially in light of the 2012 discovery of the Higgs Boson. In the future, precision measurements will be performed in order to confirm that the properties of the Higgs Boson match the Standard Model expectations.

Despite its success, the Standard Model has many inconsistencies and fails to explain many phenomena observed in nature. One of these inconsistencies is the divergence of the Higgs mass when calculated beyond leading order. This divergence is largely due to the contribution of the heavy top quark coupling to the Higgs at loop order. Several beyond Standard Model theories such as Supersymmetry (SUSY), Composite-Higgs models, and vector-like quarks offer solutions to this problem.

\footnotetext{
a e-mail: Nikolina.Ilic@ cern.ch
} 


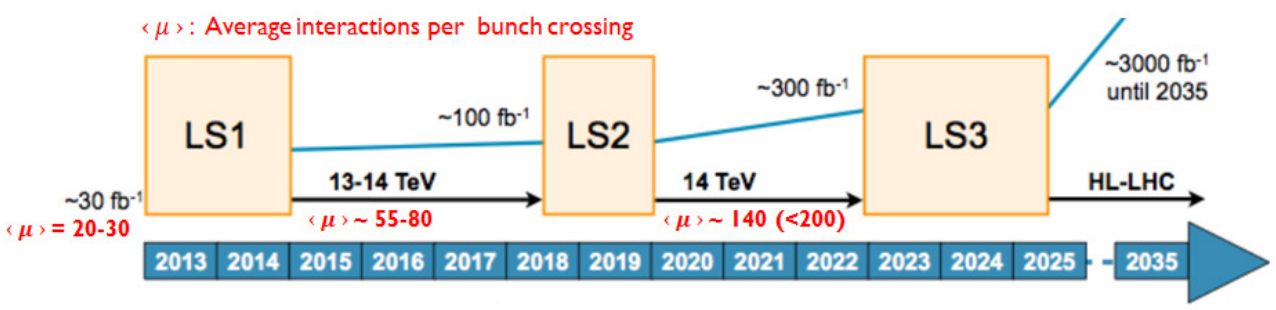

Figure 1. The plan for LHC operation, showing the planned centre-of-mass energies, total integrated luminosities, and average number of interactions per bunch crossing.

Another inconsistency within the Standard Model is that despite being theoretically possible, experimentally it is observed that Quantum Chromodynamics does not break CP symmetry. Axion models provide explanations as to why this could be the case.

Another question the Standard Model does not address is the abundance of matter with respect to anti-matter in today's universe. This conflicts with the Standard Model prediction that matter and anti-matter should have been created in equal amounts at the beginning of the universe. Beyond Standard Model Baryogenesis theories provide possible solutions to this question.

Another Standard Model inconvenience is that the electromagnetic, weak and strong coupling constants do not unify when extrapolated to higher energies. This unification is a feature of many Grand Unified Theories (GUTs), most of which include SUSY.

Finally, cosmological observations of cosmic background radiation, gravitational lensing, and red shifts of galaxies have suggested that the universe consists of 24\% Dark Matter, and 71\% Dark Energy. The failure to include Dark Matter/Energy, and gravity is one of the biggest shortcomings of the current theory. Unified field theories, such as String Theory, attempt to include all of these observations into one common framework. Many unified field theories predict extra dimensions, which suggest that gravity is weaker than other forces because it is seeping into the other dimensions.

Future searches performed in ATLAS at the upgraded LHC aim to further confirm Standard Model measurements, as well as search for deviations from the current theory. During the current LHC upgrades (LS1), the ATLAS detector is making upgrades to the tracking system. After the LHC restarts in 2015 , it will operate for three years at $\sqrt{s}=13-14 \mathrm{TeV}$, gathering $100 \mathrm{fb}^{-1}$ of data. In 2018 the LHC will shut down for upgrades (LS2), during which time ATLAS will make improvements to the trigger system in order to deal with higher trigger rates. After restarting in 2019, the LHC will operate for three years at $\sqrt{s}=14 \mathrm{TeV}$ and collect $300 \mathrm{fb}^{-1}$ of data. The final LHC shut down (LS3) will be in 2023, during which time ATLAS will install a new, higher granularity, more radiation hard inner tracker. A new triggering scheme, as well as improvements to the muon and calorimeter electronics will be made. The upgraded High-Luminosity LHC (HL LHC) will start in 2025, after which the collider is expected to collect $3000 \mathrm{fb}^{-1}$ of data. A summary of the LHC plan is shown in Figure 1.

These proceedings compare the current physics results with the results expected with $300 \mathrm{fb}^{-1}$ and $3000 \mathrm{fb}^{-1}$. The prospects for Higgs measurements are presented in section 2, searches for Supersymmetry in section 3, and exotics models such as Baryogenisis, Axion models, GUTs and ToFs in section 4. Due to the large increase in the number of interactions, one of the biggest challenges in the future will be reconstructing objects in the detector with the same accuracy. The average interactions per bunch crossing is expected to increase from $\langle\mu\rangle=20-30$ at $8 \mathrm{TeV}$ to $\langle\mu\rangle=140$ at $14 \mathrm{TeV}$. Nonetheless for the analyses presented in these proceedings, it is assumed that the detector will keep the current performance due to hardware and software upgrades. Due to changes in plans for LHC 

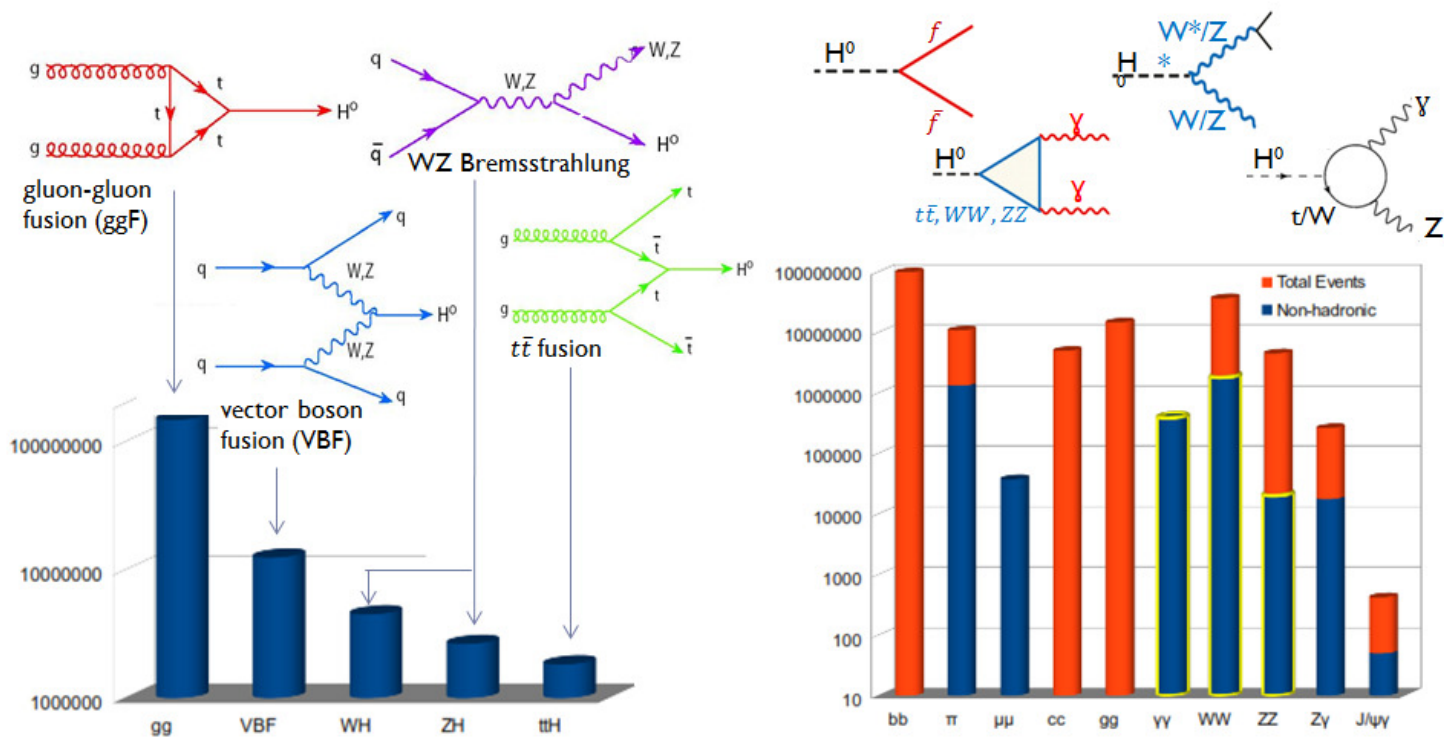

Figure 2. Left: The Higgs production modes (left) and decay modes (right) with their expected number of events at HL-LHC with $3000 \mathrm{fb}^{-1}$.

operation, the analyses were performed based on simulation of $\langle\mu\rangle=60$ for $300 \mathrm{fb}^{-1}$, instead of the currently predicted $\langle\mu\rangle=140$.

\section{Higgs Prospects}

At the LHC the Higgs, can be produced through gluon-gluon fusion, vector-boson fusion, WZ bremsstrahlung, and in associated with $t \bar{t}$, as shown by the diagrams on the left of Figure 2 . The HL-LHC is expected to produce millions of these processes, as shown by the chart in the same figure. The Higgs can decay to a fermion and anti-fermion, photons, $W / Z$ bosons and $W \gamma$ bosons as shown by the diagrams on the right of Figure 2. The same illustration shows expected number of events produced for these processes at HL-LHC.

\subsection{Improvements to Current Searches}

Despite the larger branching ratio of Higgs decaying hadronically, most 2010-2012 searches have focused on leptonic Higgs decays which are easier to separate from their backgrounds. The decay modes that have been explored are $H \rightarrow \gamma \gamma, H \rightarrow Z \rightarrow 4 \ell, H \rightarrow W W \rightarrow \ell v \ell v, H \rightarrow \tau \tau \rightarrow$ $2 \ell 4 v / \ell \tau_{\text {hadronic }}+3 v$, and $H \rightarrow b \bar{b}$. Most of these decay channels included all of the Higgs production mechanisms. The $H \rightarrow W W \rightarrow \ell v \ell v$ search excluded Higgs production in association with $t \bar{t}$, and the $H \rightarrow b \bar{b}$ channel only included $W / Z$ bremsstrahlung due to the difficulty associated in separating these two processes from other hadronic final states. The measure used in quantifying the compatibility of the data with the Standard Model Higgs hypothesis is the significance, $Z$, and signal strength, $\hat{\mu}$. The significance quantifies the excess of events in the data with respect to the background-only hypothesis. 


\begin{tabular}{|c|c|c|c|c|}
\hline Processes & Value & Current & $300 \mathrm{fb}^{-1}$ & $3000 \mathrm{fb}^{-1}$ \\
\hline$H \rightarrow \gamma \gamma$ & $\Delta \hat{\mu} / \hat{\mu}$ & $20 \%$ & - & $\leq 10 \%$ \\
\hline$H \rightarrow Z Z \rightarrow 4 \ell$ & $\Delta \hat{\mu} / \hat{\mu}$ & $27 \%$ & $15 \%$ & $13 \%$ \\
\hline$H \rightarrow W W \rightarrow \ell \nu \ell v$ & $\Delta \hat{\mu} / \hat{\mu}$ & $30 \%$ & $17 \%$ & $15 \%$ \\
\hline$H \rightarrow \tau \tau \rightarrow 2 \ell 4 v$ & $Z$ & $4.1 \sigma$ & $6.9 \sigma$ & - \\
\cline { 2 - 5 }$/ \ell \tau_{\text {had }}+3 v$ & $\Delta \hat{\mu} / \hat{\mu}$ & $31 \%$ & $15 \%$ & - \\
\hline \multirow{2}{*}{$H \rightarrow b \bar{b}$} & $Z$ & 0 & $3.9 \sigma$ & $8.8 \sigma$ \\
\cline { 2 - 5 } & $\Delta \hat{\mu} / \hat{\mu}$ & $100 \%$ & $25 \%$ & $14 \%$ \\
\hline
\end{tabular}

Table 1. A projection of the improvement in the uncertainty on $\hat{\mu}$ and significance with $300 \mathrm{fb}^{-1}$ and $3000 \mathrm{fb}^{-1}$ for all Higgs analyses completed during the 2010-2012 operational period [1], [2], [3], [4], [5].

The signal strength, shown in equation 1, quantifies the deviation of the measured cross section, $\sigma$, times branching ratio, $B R$, divided by the Standard Model prediction.

$$
\hat{\mu}=\frac{\sigma \times B R}{(\sigma \times B R)_{S M}}
$$

In all of the searches performed the signal strength is close to one, and thus consistent with the Standard Model prediction within the theoretical and experimental uncertainty. However the uncertainty on the $\hat{\mu}$ value, denoted by, $\Delta \hat{\mu} / \hat{\mu}$, is still quite large as shown in the first column of Table 1. Reducing this uncertainty with the $300 \mathrm{fb}^{-1}$ and $3000 \mathrm{fb}^{-1}$ datasets is essential in confirming the properties of the Higgs Boson and observing deviations from the Standard Model prediction. Table 1 , shows that with $300 \mathrm{fb}^{-1}$, the uncertainty will be reduced by approximately a factor of two in most of the channels due to the larger statistics. With the $3000 \mathrm{fb}^{-1}$ dataset, the improvement is somewhat smaller since the uncertainties are dominated by theoretical sources rather than due to lack of statistics. Table 1 shows that the significances in the $H \rightarrow \tau \tau$, and $H \rightarrow b \bar{b}$ will also drastically increase with a larger dataset. The significances for the $H \rightarrow \gamma \gamma, H \rightarrow Z Z$, and $H \rightarrow W W$ channels are not shown since they will be well past the discovery significance of $5 \sigma$. The large improvement in the $H \rightarrow b \bar{b}$ processes with the $3000 \mathrm{fb}^{-1}$ data is due to multi-variate analysis techniques, and predicted improvements in identifying jets originating from $b$-quarks.

\subsection{New Searches}

Along with improvements to the current Higgs analyses, several new searches will be within reach with the $3000 \mathrm{fb}^{-1}$ dataset. A search for the Higgs produced via all production modes and decaying to $\mu \mu$ final states will allow for the possibility to probe the Higgs coupling to second generation fermions for the first time. This search is expected to yield $Z=7 \sigma$ and $\Delta \hat{\mu} / \hat{\mu}=21 \%$ with the $3000 \mathrm{fb}^{-1}$ [1]. The left side of Figure 3, shows that a $H \rightarrow \mu \mu$ signal peak is distinguishable from its main $Z, t \bar{t}$ and $W W$ backgrounds with a $3000 \mathrm{fb}^{-1}$ dataset.

Another new search within reach is the production of the Higgs through gluon-gluon fusion and vector boson fusion, and its decay to $Z \gamma$, where $Z \rightarrow e e$. This final decay state is particularily interesting since it happens via a loop interaction and is thus sensitive to new particle couplings which can enter through the loop. This search is expected to obtain $Z=3.9 \sigma$ and $\Delta \hat{\mu} / \mu=30 \%$ at $3000 \mathrm{fb}^{-1}$ [6]. The right of Figure 3 shows the $H \rightarrow Z \gamma$ peak with respect to the background for $3000 \mathrm{fb}^{-1}$. 

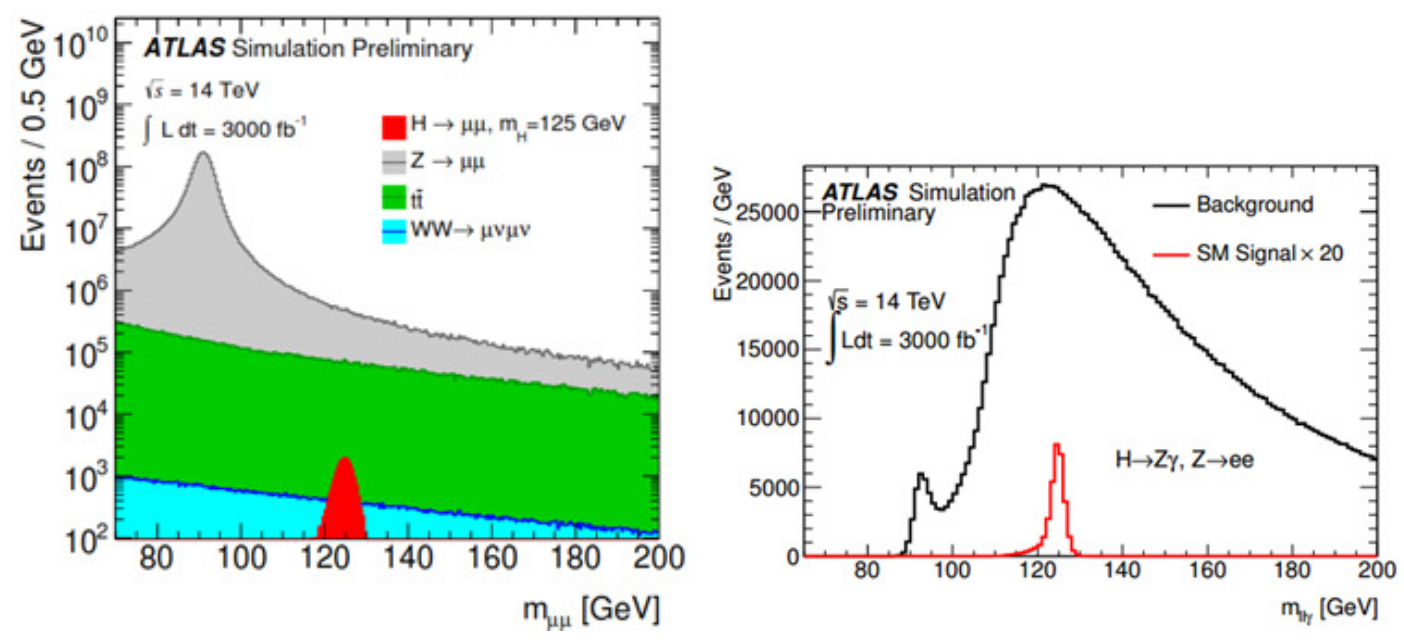

Figure 3. The signal and background shown for the $H \rightarrow \mu \mu$ search on the at $3000 \mathrm{fb}^{-1}$ (left) [1]. The signal and background for the $H \rightarrow Z \gamma, Z \rightarrow e e$ search at $3000 \mathrm{fb}-1$ (right)[6].

\subsection{Coupling Measurements}

In order to confirm that the Higgs has the properties predicted by the Standard Model, the strengths of couplings of different particles to each other are measured. The production rate of processes is proportional to their couplings squared, $g^{2}$. The measured couplings can be parametrized in terms of the expected Standard Model couplings by defining $\kappa=g / g_{S M}$, where $g$ is the measured coupling and $g_{S M}$ is the Standard Model expectation. The branching ratios and cross sections can then be expressed in terms of $\kappa$, as shown in equation 2.

$$
\frac{\sigma \cdot B R(g g \rightarrow H \rightarrow \gamma \gamma)}{\sigma_{S M}(g g \rightarrow H) \cdot B R_{S M}(H \rightarrow \gamma \gamma)}=\frac{\kappa_{g}^{2} \cdot \kappa_{\gamma}^{2}}{\kappa_{H}^{2}}
$$

In equation $2, \kappa_{H}$ is the coupling of the total Higgs width calculated from the sum of all partial widths. If no assumption is made on the total Higgs width, then it is only possible to measure the ratio of couplings, denoted by $\lambda$. Figure 2.3 shows the uncertainties on the ratio of couplings of different particles to each other. The hashed lines on the bars represent the contributions from the current theoretical uncertainties. There is a factor of three improvement in the precision of coupling measurements obtained in going from the $300 \mathrm{fb}^{-1}$ dataset, represented by the green bars, to the 3000 $\mathrm{fb}^{-1}$ dataset, represented by the blue lines. This figure demonstrates that for the $3000 \mathrm{fb}^{-1}$ dataset, it is possible to determine $\lambda$ to within 3-10\% for bosons, gluons and fermions coupling to each other. The $Z \gamma$ couplings are the only ones with larger (30\%) uncertainties. For the results used in Figure 2.3, the latest results on $H \rightarrow b \bar{b}$ were not available, and thus these inputs were fixed based on the $H \rightarrow \tau \tau$ measurement.

\section{Direct Supersymmetry Search Prospects}

Supersymmetry is a theory that provides solutions to some Standard Model problems, and explanations for beyond Standard Model phenomena. SUSY can prevent the Higgs mass from diverging 


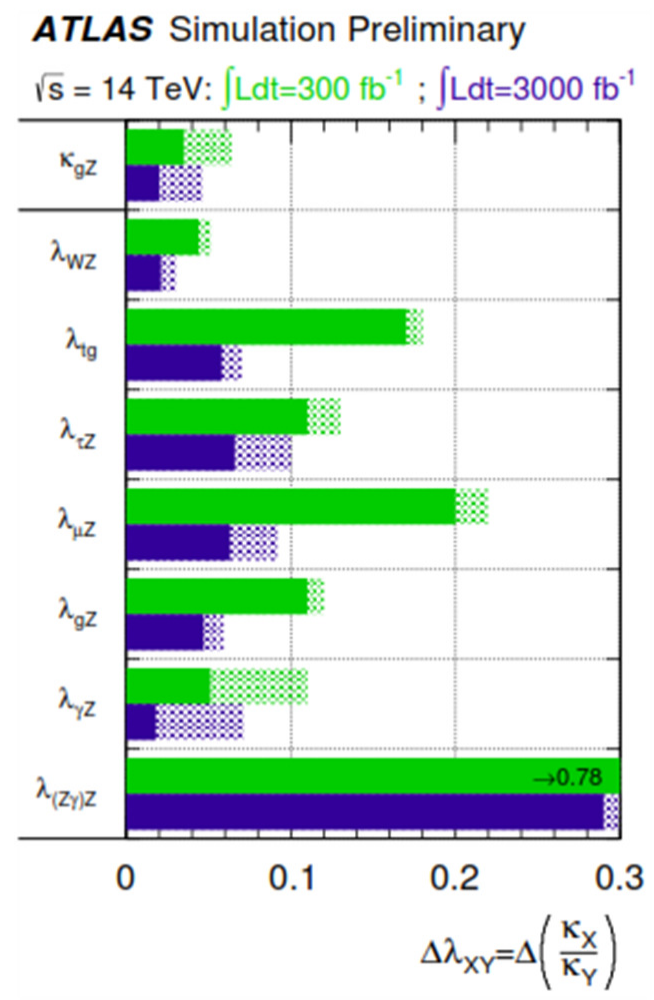

Figure 4. The uncertainties on the coupling measurements for the $300 \mathrm{fb}^{-1}$, and $3000 \mathrm{fb}^{-1}$ datasets [1].

beyond leading order, by predicting the existence of particles which cancel the loop corrections to the Higgs mass. In addition some models of SUSY provide the unification of the electromagnetic, weak and strong coupling constants at higher energy. The lightest stable SUSY particle is a candidate for Dark Matter. SUSY is also needed for many unified field theories.

SUSY predicts that for every Standard Model particle, there exists a SUSY particle (sparticle) whose spin differs by $1 / 2$. Sparticles include the partners of the quarks, denoted as squarks $(\tilde{u}, \tilde{d}, \tilde{c}, \tilde{s}, \tilde{t}, \tilde{b})$ and partners of the gluons, denoted as gluinos $(\tilde{g})$. Sparticles also include neutralinos $\left(\tilde{\chi}^{0}\right)$ and charginos $\left(\tilde{\chi}^{ \pm}\right)$which are superpositions of the Higgsino, Wino and Zino, the Higgs, $W$ and $Z$ partners. The neutralino is the lighest stable SUSY particle which interacts weakly and would leave the detector undetected.

Searches for the SUSY signal are performed by searching for the decay products of all the sparticles mentioned above. The sparticles decay to different combinations of jets, leptons, neutrinos, and neutranlinos, as shown in Figure 5. The relative production cross sections of the different sparticles at the HL-LHC with $3000 \mathrm{fb}^{-1}$ is shown on the left in Figure 6. Limit plots are made to show the $Z=5 \sigma$ discovery of each sparticle as a function of the $\tilde{\chi}^{0}$ mass versus the sparticle mass. The same limit plots, show the exclusion of the sparticle at a $95 \%$ confidence level as a function of the $\tilde{\chi}^{0}$ mass versus the sparticle mass. An example of a limit plot is shown on the right of Figure 6 . This figure shows that for a low mass $\tilde{\chi}^{0}$, the $\tilde{\chi}^{ \pm}$sparticle can be discovered if its mass is below 560 (820) $\mathrm{GeV}$, and excluded if its mass is below 840 (1100) $\mathrm{GeV}$ for the $300(3000) \mathrm{fb}^{-1}$ dataset. 


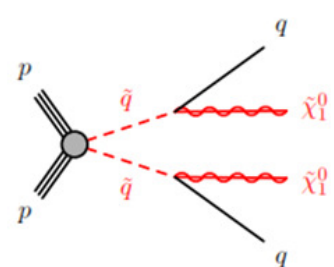

$\tilde{u}, \tilde{d}, \tilde{c}, \tilde{s}$

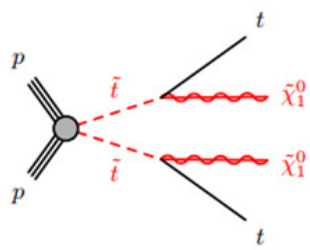

stop $(\tilde{t})$

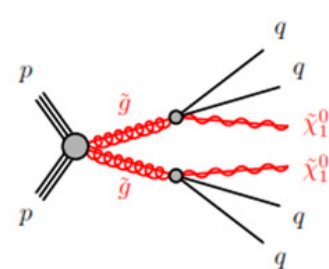

gluinos $(\tilde{g})$

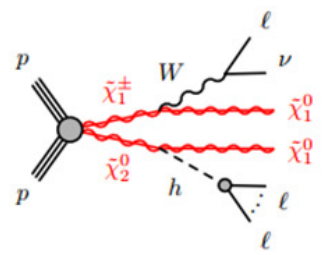

neutrolinos $\left(\tilde{\chi}^{0}\right) / \operatorname{charginos}\left(\tilde{\chi}^{ \pm}\right)$

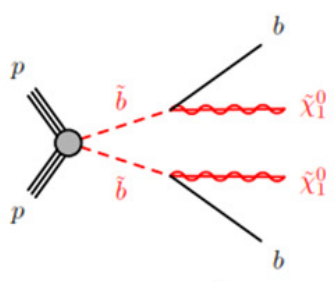

sbottom $(\tilde{b})$

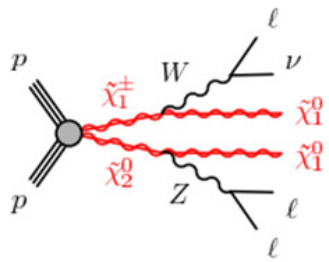

$\ell$

Figure 5. The production and final decay states of all sparticles. The chargino and neutralinos are shown when produced in association with a $W h$ and $W Z$ where $h$ is the lightest SUSY Higgs.
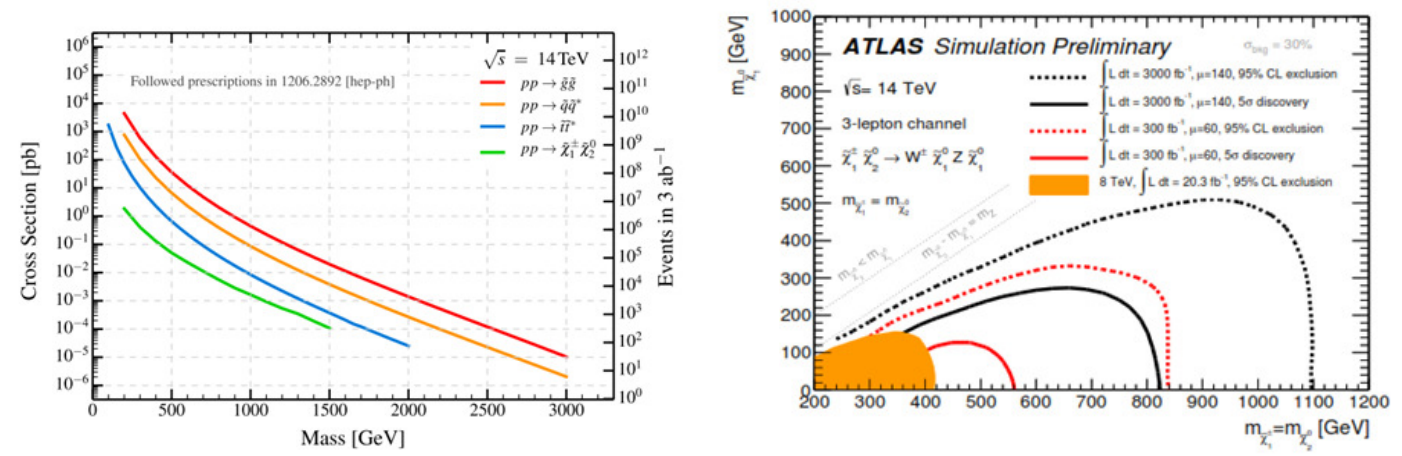

Figure 6. The cross sections of different sparticle productions for $3000 \mathrm{fb}^{-1}$ (left) [7]. The limit plot for the search for charginos (right) [7].

The discovery and exclusion limit for the remaining sparticles are summarized in Table 2. The search for the $\tilde{u}, \tilde{d}, \tilde{c}, \tilde{s}$ sparticles is performed for a scenario in which the $\tilde{g}$ is very heavy and decoupled from squarks (A), and a scenario in which the $\tilde{g}$ is around $4.5 \mathrm{TeV}$ (B). In the latter scenario squark production is enchanced. The search for the chargino and neutralino is performed when the sparticles are produced together with the $W / Z$ as well as $W h$, where $h$ is the lightest SUSY Higgs. The $W Z$, and $W h$ are searched for in leptonic decay modes. Not shown in the table is the fact that the chargino and neutralino search is also performed when the Higgs decays to a $\tau \tau$ pair, resulting in a $550 \mathrm{GeV}$ exclusion limit for $3000 \mathrm{fb}^{-1}$. 


\begin{tabular}{|c|c|c|c|c|c|}
\hline & \multicolumn{3}{|c|}{ Exclusion } & \multicolumn{2}{c|}{ Discovery } \\
\hline Sparticle & Current & $300 \mathrm{fb}^{-1}$ & $3000 \mathrm{fb}^{-1}$ & $300 \mathrm{fb}^{-1}$ & $3000 \mathrm{fb}^{-1}$ \\
\hline$\tilde{u}, \tilde{d}, \tilde{c}, \tilde{s}(\mathrm{~A})$ & 900 & 1850 & 2000 & 1300 & 1400 \\
\hline$\tilde{u}, \tilde{d}, \tilde{c}, \tilde{s}(\mathrm{~B})$ & 900 & 3100 & 3500 & 2400 & 3100 \\
\hline$\tilde{g}$ & 1400 & 2350 & 2950 & 2000 & 2350 \\
\hline$\tilde{b}$ & 600 & 1400 & 1500 & 1100 & 1300 \\
\hline$\tilde{t}$ & 650 & 1200 & 1450 & 1000 & 1200 \\
\hline$\tilde{\chi}^{0} / \tilde{\chi}^{ \pm}$via WZ & 420 & 840 & 1100 & 560 & 820 \\
\hline$\tilde{\chi}^{0} / \tilde{\chi}^{ \pm}$via $W h$ & - & 650 & 940 & - & 650 \\
\hline
\end{tabular}

Table 2. The exclusion and discovery limits on masses of sparticles, in $\mathrm{GeV}$, for the 2010-2012 data, and future $300 \mathrm{fb}^{-1}$ and $3000 \mathrm{fb}^{-1}$ datasets [7] [8].

\section{Exotics Prospects}

The current searches for exotic models which are used to explain beyond Standard Model phenomena can greatly be increased with the $300 \mathrm{fb}^{-1}$ and $3000 \mathrm{fb}^{-1}$ dataset. Evidence for Dark Matter, SUSY, Grand Unified Theories, Extra Dimensions, Axion and Baryogenesis models are searched for either through direct searches, or searches for 2 Higgs Doublet signatures, flavour changing neutral currents, and di-lepton and di-top resonances.

\subsection{Dark Matter}

Searches for Dark Matter particles offer promising improvements at the upgraded LHC. Dark matter particles can be searched for by assuming they couple to the Higgs boson. A processes that is often assumed is the production of the Higgs, in associated with a leptonically decaying $Z$, and its subsequent decay to Dark Matter. The final states of this processes include leptons and missing transverse energy from the undetected passage of Dark Matter through the detector. This analysis currently excludes such processes at a $95 \%$ confidence level if the branching ratio of this process is above $65 \%$ [9]. With the 300 (3000) $\mathrm{fb}^{-1}$ dataset, an exclusion on branching ratios above $23-32 \%(8-16 \%)$ can be reached [1]. The variation in the percentages is due to the assumption of different models for treatment of systematic uncertianties.

Direct searches for Dark Matter are also performed by assuming that Dark Matter couples to Standard Model particles via a contact interaction. The contact interaction assumes that the mass of the mediatior is much greater than the invariant mass of the Dark Matter particle. In these interactions Dark Matter is produced in associated with another object, such as a jet. The search for a high energy jet in association with missing transverse energy probes the significance as a function of the suppression scale, $M^{*}$, defined in equation 3 .

$$
M^{*}=\frac{M_{\text {mediator }}}{\sqrt{g_{S M} g_{D M}}}
$$

In equation $3, M_{\text {mediator }}$, is the mass of the mediator and $g_{S M}\left(g_{D M}\right)$ represent the couplings of the mediator to the Standard Model particle (Dark Matter). The significance plot for this search is shown on the left of Figure 4.2 for $300 \mathrm{fb}^{-1}$, assuming systematic uncertianties of $1 \%$ and $5 \%$. This results is approximately a factor of two improvement from the 2011-2012 analysis. Large improvement in this plot is not observed for the $3000 \mathrm{fb}^{-1}$ dataset because the analysis becomes systematics rather than statistics limited. 

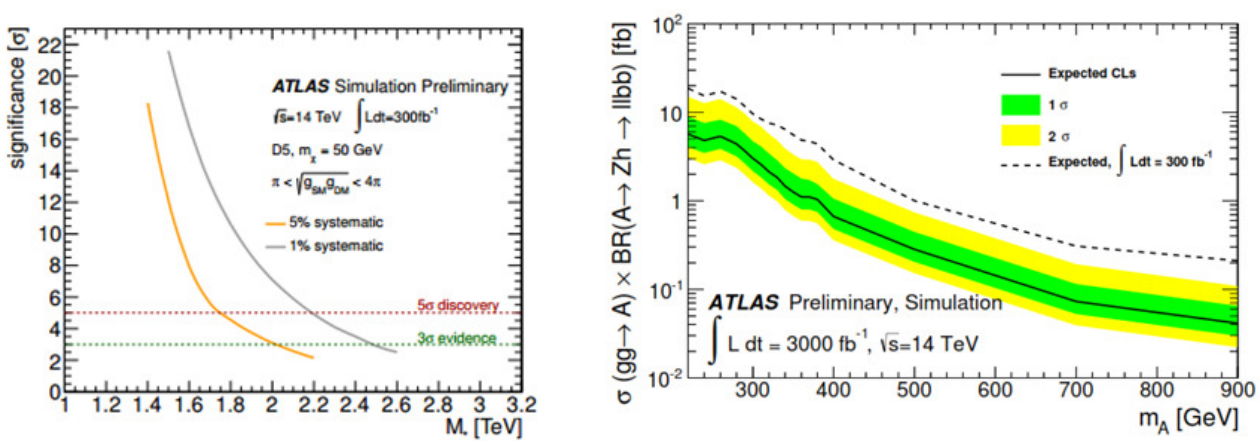

Figure 7. The significance as a function of the suppression scale right [10] (left). Limits for cross section times branching ration for gluon gluon fusion production of A, and decay to $A \rightarrow Z h, Z \rightarrow e e / \mu \mu, h \rightarrow b \bar{b}$ in $2 \mathrm{HDM}$ models (right) [11].

\subsection{Higgs Doublet Models (2HDM)}

The motivation for studying Two Higgs Doublet Models (2HDM) models is that they are included in many SUSY, Axion and Baryogenesis theories. The 2HDM models predict the existence of a complex Higgs doublet instead of the current Standard Model scalar Higgs field. This prediction results in the existence of five Higgs-like particles: the Standard Model Higgs, $h$; two heavier charged Higgs particles $H^{ \pm}$; a heavier neutral Higgs particle, $H^{0}$; a CP-odd particle, $A$. A search is performed in which the $A$ is produced via gluon-gluon fusion and decays to $Z h$ with $Z \rightarrow e e \mu \mu, h \rightarrow b \bar{b}$. The exclusion limits on the cross section times branching ratio for this process are shown in Figure 4.2 for $300 \mathrm{fb}^{-1}$. These limits improve by a factor of three for $3000 \mathrm{fb}^{-1}$.

\subsection{Flavour Changing Neutral Currents (FCNC)}

In the Standard Model, Flavour Changing neutral Currents (FCNC) are forbidden at tree level, and highly suppressed at loop level. However, this process is enhanced in several 2HDM, SUSY and extra dimension models. Thus a search is performed processes in which a top quark changes to a different flavour quark and a $Z, \gamma$ or Higgs are produced. The $t \rightarrow q Z$ search is expected to increase the exclusion limit on the branching ratio for this process from the current $0.21 \%$ to $4.1 \times 10^{-5}$ with $3000 \mathrm{fb}^{-1}$ [12]. The $t \rightarrow q \gamma$ search is expected to increase the limit from the current $3.2 \%$ to $1.3 \times 10^{-5}$ with $3000 \mathrm{fb}^{-1}$ [12]. Finally the $t \rightarrow c H$, where $H \rightarrow \gamma \gamma$ search is expected to increase the exclusion limit on the branching ratio from the current $0.83 \%$ to $1.2-1.4 \times 10^{-5}$ with $3000 \mathrm{fb}^{-1}$ [13], [14].

\subsection{Di-lepton and Di-top Resonances}

Most Grand Unified Theories predict the existence of a $Z^{\prime}$ boson decaying to $e e / \mu \mu$ final states. Since unified theories often include SUSY models, a Supersymmetric Standard Model (SSM) $Z^{\prime}$ is searched for in the di-lepton (ee/ $\mu \mu$ channel). Table 3, shows the current and future exclusion limits on the mass of a $Z^{\prime}$ boson.

In extra-dimension models and composite Higgs models, Kaluza-Klein particles, $g_{\kappa \kappa}$, are strongly produced wide resonances that decay to $t \bar{t}$. In models such as topcolour, the $Z^{\prime}$ can also decay to $t \bar{t}$. The current and future exclusion limits on the mass of such di-top resonances is also shown in Table 3. 


\begin{tabular}{|c|c|c|c|}
\hline Model & Current & $300 \mathrm{fb}^{-1}$ & $3000 \mathrm{fb}^{-1}$ \\
\hline$Z_{S S M}^{\prime} \rightarrow e e$ & 2.76 & 6.5 & 7.8 \\
\hline$Z_{S S M}^{\prime} \rightarrow \mu \mu$ & 2.53 & 6.4 & 7.6 \\
\hline$g_{\kappa \kappa} \rightarrow t \bar{t}$ & 2.0 & 4.3 & 6.7 \\
\hline$Z_{\text {topcolor }}^{\prime} \rightarrow t \bar{t}$ & 1.8 & 3.3 & 5.5 \\
\hline
\end{tabular}

Table 3. The exclusion limits on mass (in $\mathrm{TeV}$ ) of $Z^{\prime}$ and Kaluza-Klein particles decaying to di-lepton and di-top pairs [15], [16], [17].

\section{Conclusion}

The upgraded LHC running at higher centre of mass energies is expected to collect $300 \mathrm{fb}^{-1}$ before the first shutdown, and $3000 \mathrm{fb}^{-1}$ in the final phase. The uncertainty on the signal strength in the current Higgs analyses will be greatly reduced with the new data. In addition, Higgs couplings to second generation fermions and Higgs decaying via loops to $Z \gamma$ final states can be explored. The exclusion and discovery limits for SUSY particles is also dramatically increased with additional data. Finally general searches for Dark Matter, 2HDM models, flavour changing neutral currents, and dilepton/di-top resonances could provide evidence of Grand Unified Theories, extra dimensions, Axion and Baryogenesis models. The exclusion and discovery reaches for these searches are all significantly increased with additional data.

\section{References}

[1] ATLAS Collaboration, ATL-PHYS-PUB-2013-014, https://cds.cern.ch/record/1611186, (2013)

[2] ATLAS Collaboration, ATL-PHYS-PUB-2014-011, https://cds.cern.ch/record/1740962, (2014)

[3] ATLAS Collaboration, ATLAS-CONF-2013-079, https://cds.cern.ch/record/1563235, (2013)

[4] ATLAS Collaboration, ATL-PHYS-PUB-2014-012, https://cds.cern.ch/record/1741011, (2014)

[5] ATLAS Collaboration, Phys. Lett. B 726, 88 (2013)

[6] ATLAS Collaboration, ATL-PHYS-PUB-2014-006, https://cds.cern.ch/record/1703276, (2014)

[7] ATLAS Collaboration, ATL-PHYS-PUB-2014-010, https://cds.cern.ch/record/1735031, (2014)

[8] ATLAS Collaboration, ATL-PHYS-PUB-2013-011, https://cds.cern.ch/record/1604505, (2013)

[9] ATLAS Collaboration, Phys.Rev.Lett. 112, 201802 (2014), 1402 . 3244

[10] ATLAS Collaboration, ATL-PHYS-PUB-2014-007, https://cds.cern.ch/record/1708859, (2014)

[11] ATLAS Collaboration, ATL-PHYS-PUB-2013-016, https://cds.cern.ch/record/1611190, (2013)

[12] ATLAS Collaboration, ATL-PHYS-PUB-2013-007, https://cds.cern.ch/record/1564937, (2013)

[13] ATLAS Collaboration, ATLAS-CONF-2013-081, https://cds.cern.ch/record/1565103, (2013)

[14] ATLAS Collaboration, ATL-PHYS-PUB-2013-012, https://cds.cern.ch/record/1604506, (2013)

[15] ATLAS Collaboration, ATL-PHYS-PUB-2013-003, https://cds.cern.ch/record/1516108, (2013)

[16] ATLAS Collaboration, arXiv:1405.4123. CERN-PH-EP-2014-053 (2014)

[17] ATLAS Collaboration, ATLAS-CONF-2013-052, https://cds.cern.ch/record/1547568, (2013) 